\title{
SOME CURVATURE CONDITIONS ON $\alpha$-COSYMPLECTIC MANIFOLDS
}

\author{
HAKAN ÖZTÜRK \\ AFYON KOCATEPE UNIVERSITY, AFYON VOCATIONAL SCHOOL, ALI ÇETINKAYA CAMPUS, AFYONKARAHISAR, \\ TURKEY \\ ${ }^{*}$ CORRESPONDING AUTHOR: HOZTURK@AKU.EDU.TR
}

COPYRIGHT (C)2013 HORIZON RESEARCH PUBLISHING ALL RIGHTS RESERVED.

\begin{abstract}
The main interest of the present paper is to study $\alpha$-cosymplectic manifolds that satisfy some certain tensor conditions. In particular, we consider $\alpha$-cosymplectic manifolds with flatness conditions. We prove that there can not exist $\phi$-projectively flat $\alpha$-cosymplectic manifolds whose scalar curvature is zero for the dimension is greater than three. Furthermore, we work with special weakly Ricci-symmetric $\alpha$-cosymplectic manifolds. We conclude the paper with an example on $\alpha$-cosymplectic manifolds.
\end{abstract}

Keywords $\alpha$-Cosymplectic manifolds, $\alpha$-Kenmotsu manifolds, Special weakly Ricci-symmetric manifolds, Weyl conformal curvature tensor, Conharmonic curvature tensor, Projective curvature tensor

$[2000] 53 \mathrm{C} 21,53 \mathrm{C} 25$

\section{INTRODUCTION}

Tanno classified connected almost contact metric manifolds whose automorphism groups possess the maximum dimension (see [16]). The sectional curvature of plane sections for such manifolds containing the vector field $\xi$ is a constant which is called $c$. The author proved that these manifolds can be divided into three classes:

(1) Homogenous normal contact Riemannian manlfolds with $c>0$,

(2) Global Riemannian products of a line or a circle with Kaehlerian manifold whose constant holomorphic sectional curvature under the condition $c=0$.

(3) A warped product space $\mathbb{R} \times_{f} \mathbb{C}$ for the case $c<0$.

It is well known that the class (1) are characterized by admitting a Sasakian structure. Kenmotsu defined a structure closely related to the warped product which is characterized the differential geometric properties of the manifolds of class (3). The structure is known as Kenmotsu structure and in general, these structures are not Sasakian (see [8]).

Weakly symmetric and weakly Ricci-symmetric Riemannian manifolds are generalizations locally symmetric manifolds and pseudo symmetric manifold, respectively. These are manifolds in which the covariants derivative $\nabla R$ of the curvature tensor $R$ is a linear expression in $R$. The appearing coefficients of this expression are called associated 1-forms. They satisfy in the specified types of manifolds gradually weaker conditions.

Firstly, the notions of weakly symmetric and weakly Ricci-symmetric manifolds were introduced by L. Tamássy and T.Q. Binh in 1992 (see [14], [15]). In [14], the authors studied on weakly symmetric and weakly projective symmetric Riemannian manifolds. In 1993, the authors considered weakly symmetric and weakly Ricci symmetric Einstein and Sasakian manifolds (see [15]). In 2000, U. C. De, T.Q. Binh and A.A. Shaikh gave necessary conditions for the compatibility of several $K$-contact structure with weak symmetry and weakly Ricci-symmetry (see [5]). In 2002, C. Özgür, investigated weakly symmetric and weakly Ricci-symmetric Riemann-para Sasakian manifolds (see $[11])$.

The notion of special weakly Ricci symmetric manifolds was introduced and studied by H. Singh, and Q. Khan in 2001(see [13]). The authors considered special weakly symmetric manifolds. Next, Q. Khan studied some geometric properties of conharmonic Sasakian manifolds in 2004 and he also obtained some results on special weakly Ricci-symmetric Sasakian manifolds (see [10]).

In this paper, we study $\alpha$-cosymplectic manifolds with flatness conditions for $n$-dimensional case. In particular, we consider $\phi$-conformally flat, $\phi$-conharmonically flat and $\phi$-projectively flat $\alpha$-cosymplectic manifolds. We prove main results on these manifolds by using the class (3). Moreover, special weakly Ricci-symmetric $\alpha$-cosymplectic manifolds are examined. Finally, we give an example on $\alpha$-cosymplectic manifolds. 


\section{Preliminaries}

Let $\left(M^{n}, g\right)$ be an $n$-dimensional Riemannian manifold. We denote by $\nabla$ the covariant differentiation with respect to the Riemann metric $g$. Then we have

$$
R(X, Y) Z=\nabla_{X} \nabla_{Y} Z-\nabla_{Y} \nabla_{X} Z-\nabla_{[X, Y]} Z \text {. }
$$

The Riemannian curvature tensor is defined by

$$
R(X, Y, Z, W)=g(R(X, Y) Z, W) .
$$

The Ricci tensor of $M^{n}$ is defined as

$$
S(X, Y)=\operatorname{tr}\{Z \rightarrow R(X, Z) Y\}
$$

Locally, $S$ is given by

$$
S(X, Y)=\sum_{i=1}^{n} R\left(X, E_{i}, Y, E_{i}\right)
$$

where $\left\{E_{1}, E_{2}, \ldots, E_{n}\right\}$ is a local orthonormal frames field and $X, Y, Z, W$ are vector field on $M^{n}$.

The Ricci operator $Q$ is a tensor field of type $(1,1)$ on $M^{n}$ defined by

$$
g(Q X, Y)=S(X, Y)
$$

for all vector field on $M^{n}$.

Let $\left(M^{n}, g\right), n=\operatorname{dim} M, n>3$, be a connected Riemannian manifold of class $C^{\infty}$ and $\nabla$ be its Riemannian connection. The Weyl conformal curvature tensor $C$ (see [17]), the conharmonic curvature tensor $K$ (see [6]) and the projective curvature tensor $P$ (see [17]) of $\left(M^{n}, g\right)$ are defined as

$$
\begin{aligned}
& C(X, Y) Z= R(X, Y) Z-\frac{1}{n-2}[S(Y, Z) X-S(X, Z) Y \\
&+g(Y, Z) Q X-g(X, Z) Q Y \\
&+\frac{\tau}{(n-1)(n-2)}[g(Y, Z) X-g(X, Z) Y], \\
& K(X, Y) Z=\quad R(X, Y) Z-\frac{1}{n-2}[S(Y, Z) X-S(X, Z) Y \\
&+g(Y, Z) Q X-g(X, Z) Q Y, \\
& P(X, Y) Z=R(X, Y) Z-\frac{1}{n-1}[g(Y, Z) Q X-g(X, Z) Q Y],
\end{aligned}
$$

respectively, where $Q$ is the Ricci operator, $S$ is the Ricci tensor, $\tau=\operatorname{tr}(S)$ is the scalar curvature and $X, Y, Z \in$ $\chi\left(M^{n}\right), \chi\left(M^{n}\right)$ being the Lie algebra of vector fields of $M^{n}$.

Let $C$ be the Weyl conformal curvature tensor of $M^{n}$. Since at each point $p \in M^{n}$ the tangent space $T_{P}\left(M^{n}\right)$ can be decomposed into the direct sum $T_{p}\left(M^{n}\right)=\phi\left(T_{p}\left(M^{n}\right)\right) \oplus L\left(\xi_{p}\right)$, where $L\left(\xi_{p}\right)$ is a 1-dimensional linear subspace of $T_{p}\left(M^{n}\right)$ generated by $\xi_{p}$, we have a map:

$$
C: T_{p}\left(M^{n}\right) \times T_{p}\left(M^{n}\right) \times T_{p}\left(M^{n}\right) \rightarrow \phi\left(T_{p}\left(M^{n}\right)\right) \oplus L\left(\xi_{p}\right) .
$$

It may be natural to consider the following particular cases:

(1) $C: T_{p}\left(M^{n}\right) \times T_{p}\left(M^{n}\right) \times T_{p}\left(M^{n}\right) \rightarrow L\left(\xi_{p}\right)$, that is, the projection of the image of $C$ in $\phi\left(T_{p}\left(M^{n}\right)\right)$ is zero.

(2) $C: T_{p}\left(M^{n}\right) \times T_{p}\left(M^{n}\right) \times T_{p}\left(M^{n}\right) \rightarrow \phi\left(T_{p}\left(M^{n}\right)\right)$, that is, the projection of the image of $C$ in $L\left(\xi_{p}\right)$ is zero.

(3) $C: \phi\left(T_{p}\left(M^{n}\right)\right) \times \phi\left(T_{p}\left(M^{n}\right)\right) \times \phi\left(T_{p}\left(M^{n}\right)\right) \rightarrow L\left(\xi_{p}\right)$, that is, when $C$ is restricted to $\left(T_{p}\left(M^{n}\right)\right) \times \phi\left(T_{p}\left(M^{n}\right)\right) \times$ $\phi\left(T_{p}\left(M^{n}\right)\right)$, the projection of the image of in $\phi\left(T_{p}\left(M^{n}\right)\right)$ is zero. This condition is equivalent to

$$
\phi^{2} C(\phi X, \phi Y) \phi Z=0
$$

$($ see $[4])$.

A differentiable manifold $\left(M^{n}, g\right), n>3$, satisfying the condition (2.4) is called $\phi$-conformally flat.

A differentiable manifold $\left(M^{n}, g\right), n>3$, satisfying the condition

$$
\phi^{2} K(\phi X, \phi Y) \phi Z=0
$$

is called $\phi$-conharmonically flat.

A differentiable manifold $\left(M^{n}, g\right), n>3$, satisfying the condition

$$
\phi^{2} P(\phi X, \phi Y) \phi Z=0
$$

is called $\phi$-projectively flat.

The cases (1) and (2) were considered in [18] and [19], respectively. The case (3) was considered in [4] for the case $M^{n}$ is a $K$-contact manifold. In [2], the authors considered $(k, \mu)$-contact manifolds satisfying Eq. (2.5). Furthermore in [1], the authors studied $(k, \mu)$-contact metric manifolds satisfying Eq. (2.4).

In [12], the author proves that an $n$-dimensional $(n>3)$ conformally flat Lorentzian para-Sasakian manifold is an $\eta$-Einstein manifold, conharmonically flat Lorentzian para-Sasakian manifold is an Einstein manifold with zero 
scalar curvature. Also the author showed that a projectively flat Lorentzian para-Sasakian manifold is an Einstein manifold with scalar curvature $\tau=n(n-1)$.

\section{3. $\alpha$-Cosymplectic Manifolds}

Let $M^{n}$ be an $n$-dimensional differentiable manifold equipped with a triple $(\phi, \xi, \eta)$, where $\phi$ is a $(1,1)$-tensor field, $\xi$ is a vector field, $\eta$ is a 1 -form on $M^{n}$ such that

$$
\eta(\xi)=1, \quad \phi^{2}=-I+\eta \otimes \xi
$$

which implies

$$
\phi \xi=0 \quad \eta \circ \phi=0 \quad \operatorname{rank}(\phi)=n-1 .
$$

If $M^{n}$ admits a Riemannian metric $g$, such that

$$
g(\phi X, \phi Y)=g(X, Y)-\eta(X) \eta(Y), \eta(X)=g(X, \xi)
$$

then $M^{n}$ is said to admit almost contact structure $(\phi, \xi, \eta, g)$.

On such a manifold, the fundamental $\Phi$ of $M^{n}$ is defined as

$$
\Phi(X, Y)=g(\phi X, Y),, X, Y \in \Gamma(T M) .
$$

An almost contact metric manifold $(M, \phi, \xi, \eta, g)$ is said to be almost cosymplectic if $d \eta=0$ and $d \Phi=0$, where $d$ is the exterior differential operator. The products of almost Kaehlerian manifolds and the real line $\mathbb{R}$ or the $S^{1}$ circle are the simplest examples of almost cosymplectic manifolds. An almost contact manifold $(M, \phi, \xi, \eta)$ is said to be normal if the Nijenhuis torsion

$$
N_{\phi}(X, Y)=[\phi X, \phi Y]-\phi[\phi X, Y]-\phi[X, \phi Y]+\phi^{2}[X, Y]+2 d \eta(X, Y) \xi
$$

vanishes for any vector fields $X$ and $Y$. A normal almost cosymplectic manifolds is called a cosymplectic manifold. As it is known that an almost contact metric structure is cosymplectic if and only if both $\nabla \eta$ and $\nabla \Phi$ vanish.

An almost contact metric manifold $M^{n}$ is said to be almost $\alpha$-Kenmotsu if $d \eta=0$ and $d \Phi=2 \alpha \eta \wedge \Phi, \alpha$ being a non-zero real constant. It is worthwhile to note that almost $\alpha$-Kenmotsu structures are related to some special local conformal deformations of almost cosymplectic structures.

In order to treat these two classes in a unified way, we have a new notion of an almost $\alpha$-cosymplectic manifold for any real number $\alpha$ that is defined as in the formula

$$
d \eta=0,, d \Phi=2 \alpha \eta \wedge \Phi .
$$

A normal almost $\alpha$-cosymplectic manifold is called an $\alpha$-cosymplectic manifold. An $\alpha$-cosymplectic manifold is either cosymplectic under the condition $\alpha=0$ or $\alpha$-Kenmotsu under the condition $\alpha \neq 0$ for $\alpha \in \mathbb{R}$.

On such an $\alpha$-cosymplectic manifold, we have

$$
\left.\left(\nabla_{X} \phi\right) Y=\alpha[g(\phi X, Y) \xi-\eta(Y) \phi X]\right)
$$

for $\alpha \in \mathbb{R}$ on $M^{n}$.

Let $M$ be a $n$-dimensional $\alpha$-cosymplectic manifold. From Eq. (3.4), it is easy to see that

$$
\nabla_{X} \xi=-\alpha \phi^{2} X
$$

where $\nabla$ denotes the Riemannian connection.

In an $\alpha$-cosymplectic manifold $M^{n}$, the following relations are held

$$
\begin{gathered}
R(\xi, X) Y=\alpha^{2}[\eta(Y) X-g(X, Y) \xi], R(X, Y) \xi=\alpha^{2}[\eta(X) Y-\eta(Y) X], \\
S(\xi, X)=-\alpha^{2}(n-1) \eta(X),
\end{gathered}
$$

$$
\begin{gathered}
S(\phi X, \phi Y)=S(X, Y)+\alpha^{2}(n-1) \eta(X) \eta(Y), \\
R(\xi, X) \xi=\alpha^{2}[X-\eta(X) \xi]=-\alpha^{2} \phi^{2} X \\
g(R(\xi, X) Y, \xi)=\alpha^{2}[\eta(X) \eta(Y)-g(X, Y]) \\
Q \xi=-\alpha^{2}(n-1) \xi \\
S(\xi, \xi)=-\alpha^{2}(n-1)
\end{gathered}
$$

for any vector fields $X, Y$ and $\alpha \in \mathbb{R}$. Kenmotsu manifolds have been studied by author [7] and he obtained the above results for $\alpha=1$.

An $\alpha$-cosymplectic manifold $M^{n}$ is said to be Einstein if its Ricci tensor $S$ is of the form

$$
S(X, Y)=\lambda g(X, Y),
$$

where $\lambda$ is constant and it is called $\eta$-Einstein if its Ricci tensor $S$ is of the form

$$
S(X, Y)=\lambda_{1} g(X, Y)+\lambda_{2} \eta(X) \eta(Y),
$$

for any vector fields $X$ and $Y$, where $\lambda_{1}$ and $\lambda_{2}$ are functions on $M^{n}$ (see [3], [17]). 


\section{Some Tensor Conditions on $\alpha$-Cosymplectic manifolds}

Theorem 1. Let $M^{n}$ be an $\alpha$-cosymplectic manifold. If the manifold $M^{n}$ is conharmonically flat Einstein manifold, then $M^{n}$ is a manifold of constant curvature such that $\alpha^{2}$ is constant.

Proof. We suppose that $M^{n}$ be an $\alpha$-cosymplectic manifold satisfying following condition

$$
K(X, Y) Z=0 .
$$

Then it follows from Eq.(2.2)

$$
\begin{aligned}
R(X, Y) Z= & \frac{1}{n-2}[S(Y, Z) X-S(X, Z) Y \\
& +g(Y, Z) Q(X)-g(X, Z) Q(Y)]
\end{aligned}
$$

Let the manifold be Einstein. Then Eq.(4.2) reduces to

or

$$
R(X, Y) Z=\frac{2 \lambda}{n-2}[g(Y, Z) X-g(X, Z) Y] .
$$

Taking $X=W=\xi$ in Eq.(4.4), then we get

$$
g(R(\xi, Y) Z, \xi)=\frac{2 \lambda}{n-2}[g(Y, Z)-\eta(Y) \eta(Z)] .
$$

Using Eq.(4.5) with the help of Eq.(3.9), we obtain

$$
\left(\frac{2 \lambda}{n-2}+\alpha^{2}\right)[g(Y, Z)-\eta(Y) \eta(Z)]=0
$$

We observe that if $g(Y, Z)-\eta(Y) \eta(Z)=0$, then $g(\phi Y, \phi Z)=0$ can be obtained by using Eq.(3.3) which is a contradiction on this structure. So it must be $\left(\frac{2 \lambda}{n-2}+\alpha^{2}\right)=0$. It follows that the manifold $M^{n}$ is cosymplectic and has zero constant curvature for $\lambda=0$. Otherwise we find $\lambda=\frac{(2-n) \alpha^{2}}{2}$ with $\alpha \neq 0$. Hence, $\alpha$-Kenmotsu case has a constant curvature such that $\alpha^{2}$ is constant. Consequently, the manifold $M^{n}$ has a constant curvature satisfying $\alpha^{2}$ is constant. As a special case, if we choose $\alpha=1$, we obtain that a conharmonically $\alpha$-cosymplectic manifold is locally isometric with a unit sphere which is proved in [10].

Definition 1. An n-dimensional Riemannian manifold $\left(M^{n}, g\right)$ is called a special weakly Ricci-symmetric manifold if

$$
\left(D_{X} S\right)(Y, Z)=2 \zeta(X) S(Y, Z)+\zeta(Y) S(X, Z)+\zeta(Z) S(Y, X)
$$

where $\zeta$ is a 1 -form and is defined as

$$
\zeta(X)=g(X, \rho)
$$

where $\rho$ is the associated vector field.

Then we can give the following result:

Theorem 2. If a special weakly Ricci-symmetric $\alpha$-cosymplectic manifold admits a cyclic Ricci tensor, then the 1 -form $\zeta$ can not be vanished.

Proof. Let Eqs.(4.6) and (4.7) be satisfied in an $\alpha$-cosymplectic manifold $M^{n}$. Taking cyclic sum of Eq.(4.6), we have

$$
\begin{aligned}
& \left(D_{X} S\right)(Y, Z)+\left(D_{Y} S\right)(Z, X)+\left(D_{Z} S\right)(X, Y) \\
& \quad=4[\zeta(X) S(Y, Z)+\zeta(Y) S(X, Z)+\zeta(Z) S(Y, X)] .
\end{aligned}
$$

Let $M$ admit a cyclic Ricci tensor. Then Eq.(4.8) reduces to

$$
\zeta(X) S(Y, Z)+\zeta(Y) S(X, Z)+\zeta(Z) S(Y, X)=0 .
$$

Taking $Z=\xi$ in Eq.(4.9), we have

$$
\zeta(X) S(Y, \xi)+\zeta(Y) S(X, \xi)+\eta(\rho) S(Y, X)=0,
$$

also taking $Y=\xi$ in Eq.(4.10) we get

$$
\zeta(X) S(\xi, \xi)+\eta(\rho) S(X, \xi)+\eta(\rho) S(\xi, X)=0 .
$$

Using $X=\xi$ in Eqs.(4.11) and (3.11), we find

$$
-3 \alpha^{2}(n-1) \eta(\rho)=0
$$

and

$$
\eta(\rho)=0 .
$$


The use of Eq.(4.13) in Eq.(4.11) gives $\zeta(X)=0, \forall X \in T(M)$. At this point we recall that 1-form $\zeta$ must be vanished for $\alpha$-Kenmotsu case. Obviously, the cosymplectic case requires that 1 -form $\zeta$ can not be vanished. It shows that 1 -form $\zeta$ need not to be vanished for $\alpha=0$. This completes the proof.

Theorem 3. A special weakly Ricci-symmetric $\alpha$-cosymplectic manifold can not be an Einstein manifold if the 1 -form $\zeta$ is equal to zero.

Proof. Since $\alpha$-cosymplectic manifold is an Einstein manifold, it holds $\left(D_{X} S\right)(Y, Z)=0$ and $S(Y, Z)=\lambda g(Y, Z)$. Hence, Eq.(4.6) gives

$$
2 \zeta(X) S(Y, Z)+\zeta(Y) S(X, Z)+\zeta(Z) S(Y, X)=0
$$

Putting $Z=\xi$ in Eq.(4.14), we get

$$
2 \zeta(X) S(Y, \xi)+\zeta(Y) S(X, \xi)+\eta(\rho) S(Y, X)=0 .
$$

Further, taking $X=\xi$ in Eq.(4.15), we have

$$
2 \eta(\rho) S(Y, \xi)+\zeta(Y) S(\xi, \xi)+\eta(\rho) S(Y, \xi)=0 .
$$

Putting $Y=\xi$ in Eq.(4.16) and with the help of Eqs.(3.1) and (3.11) provides

$$
-4 \alpha^{2}(n-1) \eta(\rho)=0
$$

In view of Eq.(4.17) in Eq.(4.16), we find $\zeta(Y)=0, \forall Y \in T(M)$ for $\alpha^{2} \neq 0$. On the other hand, cosymplectic case means that the 1 -form $\zeta$ need not be zero, which completes the proof.

\section{5. $\alpha$-Cosymplectic MANifolds With FlATNESS CONDitions}

In this section, we consider $\phi$-conformally flat, $\phi$-conharmonically flat and $\phi$-projectively flat $\alpha$-cosymplectic manifolds. According to these statements, the following results are held under certain flatness conditions.

Theorem 4. Let $M^{n}$ be an $n$-dimensional, $(n>3)$, $\phi$-conformally flat $\alpha$-cosymplectic manifold. Then $M^{n}$ is an $\eta$-Einstein manifold.

Proof. Suppose that $\left(M^{n}, g\right), n>3$, be a $\phi$-conformally flat $\alpha$-cosymplectic manifold. It is easy to see that $\phi^{2} C(\phi X, \phi Y) \phi Z=0$ holds if and only if

$$
g(C(\phi X, \phi Y) \phi Z, \phi W)=0,
$$

for any $X, Y, Z, W \in \chi\left(M^{n}\right)$. So by the use of Eq.(2.1) $\phi$-conformally flat means

$$
\begin{aligned}
g(R(\phi X, \phi Y) \phi Z, \phi W)= & \frac{1}{n-2}[g(\phi Y, \phi Z) S(\phi X, \phi W) \\
& -g(\phi X, \phi Z) S(\phi Y, \phi W)+g(\phi X, \phi W) S(\phi Y, \phi Z) \\
& -g(\phi Y, \phi W) S(\phi X, \phi Z)] \\
& -\frac{\tau}{(n-1)(n-2)}[g(\phi Y, \phi Z) g(\phi X, \phi W) \\
& -g(\phi X, \phi Z) g(\phi Y, \phi W) .
\end{aligned}
$$

Let $\left\{E_{1}, \ldots, E_{n-1}, \xi\right\}$ be a local orthonormal basis of vector fields in $M^{n}$. Using that $\left\{\phi E_{1}, \ldots, \phi E_{n-1}, \xi\right\}$ is also a local orthonormal basis, if we put $X=W=E_{i}$ in Eq.(4.2) and sum up with respect to $i$, then

$$
\begin{aligned}
\sum_{i=1}^{n-1} g\left(R\left(\phi E_{i}, \phi Y\right) \phi Z, \phi E_{i}\right)= & \left(\frac{1}{n-2}\right) \sum_{i=1}^{n-1}\left[g(\phi Y, \phi Z) S\left(\phi E_{i}, \phi E_{i}\right)\right. \\
& -g\left(\phi E_{i}, \phi Z\right) S\left(\phi Y, \phi E_{i}\right)+g\left(\phi E_{i}, \phi E_{i}\right) S(\phi Y, \phi Z) \\
& \left.-g\left(\phi Y, \phi E_{i}\right) S\left(\phi E_{i}, \phi Z\right)\right] \\
& -\left(\frac{\tau}{(n-1)(n-2)}\right) \sum_{i=1}^{n-1}\left[g(\phi Y, \phi Z) g\left(\phi E_{i}, \phi E_{i}\right)\right. \\
& -g\left(\phi E_{i}, \phi Z\right) g\left(\phi Y, \phi E_{i}\right) .
\end{aligned}
$$

It can be verify that

$$
\sum_{i=1}^{n-1} g\left(R\left(\phi E_{i}, \phi Y\right) \phi Z, \phi E_{i}\right)=S(\phi Y, \phi Z)+\alpha^{2} g(\phi Y, \phi Z)
$$

$$
\sum_{i=1}^{n-1} S\left(\phi E_{i}, \phi E_{i}\right)=\tau+\alpha^{2}(n-1)
$$

$$
\sum_{i=1}^{n-1} g\left(\phi E_{i}, \phi Z\right) S\left(\phi Y, \phi E_{i}\right)=S(\phi Y, \phi Z)
$$




$$
\sum_{i=1}^{n-1} g\left(\phi E_{i}, \phi E_{i}\right)=n-1
$$

and

$$
\sum_{i=1}^{n-1} g\left(\phi E_{i}, \phi Z\right) g\left(\phi Y, \phi E_{i}\right)=g(\phi Y, \phi Z) .
$$

So by virtue of Eqs.(4.4) and (4.8), Eq.(4.3) can be written as

$$
S(\phi Y, \phi Z)=\left(\frac{\tau}{n-1}+\alpha^{2}\right) g(\phi Y, \phi Z) .
$$

Then by making use of Eqs.(3.4) and (3.8), Eq.(4.9) takes the form

$$
S(Y, Z)=\left(\frac{\tau}{n-1}+\alpha^{2}\right) g(Y, Z)-\left(\frac{\tau}{n-1}+\alpha^{2} n\right) \eta(Y) \eta(Z)
$$

which implies that $M^{n}$ is an $\eta$-Einstein manifold by virtue of Eq.(3.12). This completes the proof.

Theorem 5. Let $M^{n}$ be an $n$-dimensional, $(n>3)$, $\alpha$-cosymplectic manifold. There can not exist $\phi$-projectively flat $\alpha$-cosymplectic manifolds with zero scalar curvature.

Proof. We assume that $M^{n}$ be an $n$-dimensional, $(n>3), \phi$-projectively flat $\alpha$-cosymplectic manifold. It can be easily seen that $\phi^{2} P(\phi X, \phi Y) \phi Z=0$ holds if and only if

$$
g(R(\phi X, \phi Y) \phi Z, \phi W)=0,
$$

for any $X, Y, Z, W \in \chi\left(M^{n}\right)$. From Eqs.(2.3) and (3.8), $\phi$-projectively flat means

$$
g(R(\phi X, \phi Y) \phi Z, \phi W)=\frac{1}{n-2}[g(\phi Y, \phi Z) S(\phi X, \phi W)-g(\phi X, \phi Z) S(\phi Y, \phi W) .
$$

Choosing $\left\{E_{1}, \ldots, E_{n-1}, \xi\right\}$ as a local orthonormal basis of vector fields in $M^{n}$ and using the fact that $\left\{\phi E_{1}, \ldots, \phi E_{n-1}, \xi\right\}$ is also a local orthonormal basis, putting $X=W=E_{i}$ in Eq.(5.10) and summing up with respect to $i$, then we have

$$
\sum_{i=1}^{n-1} g\left(R\left(\phi E_{i}, \phi Y\right) \phi Z, \phi E_{i}\right)=\frac{1}{n-2} \sum_{i=1}^{n-1}\left[g(\phi Y, \phi Z) S\left(\phi E_{i}, \phi E_{i}\right)-g\left(\phi E_{i}, \phi Z\right) S\left(\phi Y, \phi E_{i}\right) .\right.
$$

Then applying Eqs.(4.4) and (4.6) into Eq.(5.11) gives

$$
S(\phi Y, \phi Z)=\left(\frac{\tau}{n}\right) g(\phi Y, \phi Z) .
$$

By virtue of Eqs.(3.4) and (3.8), we find

$$
S(Y, Z)=\left(\frac{\tau}{n}\right) g(Y, Z)-\left(\frac{\tau}{n}+\alpha^{2}(n-1)\right) \eta(Y) \eta(Z),
$$

and contracting the above identity gives

$$
\tau+\alpha^{2} n(n-1)=0 .
$$

The condition $\alpha=0$ implies that $\tau=0$. But, $\alpha$-Kenmotsu case means $n=0$ and $n=1$, which is a contraction. That is, there can not exist $\phi$-projectively flat $\alpha$-cosymplectic manifolds with $\tau=0$.

Theorem 6. Let $M^{n}$ be an $n$-dimensional, $(n>3)$, $\phi$-conharmonically flat $\alpha$-cosymplectic manifold. Then $M^{n}$ is an $\eta$-Einstein manifold with zero scalar curvature.

Proof. We suppose that $\left(M^{n}, g\right),(n>3)$, be a $\phi$-conformally flat $\alpha$-cosymplectic manifold. It obvious that $\phi^{2} K(\phi X, \phi Y) \phi Z=0$ holds if and only if

$$
g(K(\phi X, \phi Y) \phi Z, \phi W)=0,
$$

for any $X, Y, Z, W \in \chi\left(M^{n}\right)$. Using Eq.(2.2), $\phi$-conformally flat gives

$$
\begin{aligned}
g(R(\phi X, \phi Y) \phi Z, \phi W)= & \left(\frac{1}{n-2}\right)[g(\phi Y, \phi Z) S(\phi X, \phi W) \\
& -g(\phi X, \phi Z) S(\phi Y, \phi W)+g(\phi X, \phi W) S(\phi Y, \phi Z) \\
& -g(\phi Y, \phi W) S(\phi X, \phi Z)] .
\end{aligned}
$$

Similar to the proof of above theorem, we can suppose that $\left\{E_{1}, \ldots, E_{n-1}, \xi\right\}$ is a local orthonormal basis of vector fields in $M^{n}$. By using the fact that $\left\{\phi E_{1}, \ldots, \phi E_{n-1}, \xi\right\}$ is also a local orthonormal basis, if we put $X=W=E_{i}$ 
in Eq.(4.13) and sum up with respect to $i$, then

$$
\begin{aligned}
\sum_{i=1}^{n-1} g\left(R\left(\phi E_{i}, \phi Y\right) \phi Z, \phi E_{i}\right)= & \frac{1}{n-2} \sum_{i=1}^{n-1}\left[g(\phi Y, \phi Z) S\left(\phi E_{i}, \phi E_{i}\right)\right. \\
& -g\left(\phi E_{i}, \phi Z\right) S\left(\phi Y, \phi E_{i}\right)+g\left(\phi E_{i}, \phi E_{i}\right) S(\phi Y, \phi Z) \\
& \left.-g\left(\phi Y, \phi E_{i}\right) S\left(\phi E_{i}, \phi Z\right)\right] .
\end{aligned}
$$

Make use of Eqs.(4.4) and (4.7), Eq.(4.14) turns into

$$
S(\phi Y, \phi Z)=\left(\tau+\alpha^{2}\right) g(\phi Y, \phi Z) .
$$

Then applying Eqs.(3.4) and (3.8) into Eq.(4.15) we have

$$
S(Y, Z)=\left(\tau+\alpha^{2}\right) g(Y, Z)-\left(\tau+\alpha^{2} n\right) \eta(Y) \eta(Z),
$$

that is, $M^{n}$ is an $\eta$-Einstein manifold. By contracting Eq.(4.16) we find $(2-n) \tau=0$, which implies the scalar curvature $\tau=0$, completing the proof.

\section{Discussion}

It is well known that $\alpha$-cosymplectic manifolds can be derived from almost contact Riemannian manifolds. Many different types of almost contact structures are defined in the literature (almost cosymplectic, cosymplectic, almost $\alpha$-Kenmotsu, $\alpha$-Kenmotsu,..., [8], [16]). Many tensor conditions are valid for these types of manifolds. In particular, we examine $\alpha$-cosymplectic structures which have some certain tensor conditions. We have known that an almost contact metric manifold is said to be normal providing that the Nijenhuis torsion vanishes for any vector fields on these kind of manifolds. Curvature properties of almost $\alpha$-cosymplectic manifolds are more complicated than $\alpha$-cosymplectic manifolds. Hence, the calculation difficulties are frequently encountered by the authors. Although these manifolds possess residual statements, obtained results are more attractive and larger. Therefore, general results can be achieved by using deformations and some certain conditions.

The notion of almost $\alpha$-cosymplectic manifolds was introduced by Kim and Pak, where $\alpha$ is a scalar (see [9]). But it need not be constant. In this paper, we consider that $\alpha$ is a real constant $(\alpha \in \mathbb{R})$. We want to generalize these manifolds which have certain tensor properties by choosing a real-valued function $\alpha$, where $\alpha$ is a smooth function on almost $\alpha$-cosymplectic manifold satisfying the condition $d \alpha \wedge \eta=0$. Our forthcoming papers will be dedicated to this topic.

Example 1. We assume the 3 -dimensional manifold $M^{3}=\left\{\left(x_{1}, x_{2}, x_{3}\right) \in \mathbb{R}^{3}, x_{3} \neq 0\right\}$, where $\left(x_{1}, x_{2}, x_{3}\right)$ are the standard coordinates in $\mathbb{R}^{3}$. The vector fields are

$$
\begin{aligned}
& e_{1}=e^{\alpha x_{3}} \frac{\partial}{\partial x_{1}}, \\
& e_{2}=e^{\alpha x_{3}} \frac{\partial}{\partial x_{2}}, \\
& e_{3}=\frac{\partial}{\partial x_{3}},
\end{aligned}
$$

where $\alpha \in \mathbb{R}$. It is obvious that $\left\{e_{1}, e_{2}, e_{3}\right\}$ are linearly independent at each point of $M^{3}$. Let $g$ be the Riemannian metric defined by

$$
g\left(e_{1}, e_{1}\right)=g\left(e_{2}, e_{2}\right)=g\left(e_{3}, e_{3}\right)=1, g\left(e_{1}, e_{2}\right)=g\left(e_{1}, e_{3}\right)=g\left(e_{2}, e_{3}\right)=0
$$

and given by the tensor product

$$
g=\frac{1}{e^{2 \alpha x_{3}}}\left(d x_{1} \otimes d x_{1}+d x_{2} \otimes d x_{2}\right)+d x_{3} \otimes d x_{3} .
$$

Let $\eta$ be the 1 -form defined by $\eta(X)=g\left(X, e_{3}\right)$ for any vector field $X$ on $M^{3}$ and $\phi$ be the $(1,1)$ tensor field defined by

$$
\phi\left(e_{1}\right)=e_{2}, \phi\left(e_{2}\right)=-e_{1}, \phi\left(e_{3}\right)=0 .
$$

Then using linearity of $g$ and $\phi$, we have

$$
\phi^{2} X=-X+\eta(X) e_{3}, \eta\left(e_{3}\right)=1, g(\phi X, \phi Y)=g(X, Y)-\eta(X) \eta(Y),
$$

for any vector fields on $M^{3}$.

Let $\nabla$ be the Levi-Civita connection with respect to the metric $g$. Then we get

$$
\left[e_{1}, e_{3}\right]=\alpha e_{1},\left[e_{2}, e_{3}\right]=\alpha e_{2},\left[e_{1}, e_{2}\right]=0 .
$$

Using Koszul's formula, the Riemannian connection $\nabla$ of the metric $g$ is given by

$$
\begin{aligned}
2 g\left(\nabla_{X} Y, Z\right)= & X g(Y, Z)+Y g(Z, X)-Z g(X, Y) \\
& -g(X,[Y, Z])-g(Y,[X, Z])-g(Z,[X, Y]) .
\end{aligned}
$$


Koszul's formula yields

$$
\begin{aligned}
& \nabla_{e_{1}} e_{1}=-\alpha e_{3}, \quad \nabla_{e_{1}} e_{2}=-e_{3}, \quad \nabla_{e_{1}} e_{3}=\alpha e_{1} \\
& \nabla_{e_{2}} e_{1}=-e_{3}, \quad \nabla_{e_{2}} e_{2}=-\alpha e_{3}, \quad \nabla_{e_{2}} e_{3}=\alpha e_{2}, \\
& \nabla_{e_{3}} e_{1}=0, \quad \nabla_{e_{3}} e_{2}=0, \quad \nabla_{e_{3}} e_{3}=0 .
\end{aligned}
$$

In addition, one can easily obtain by simple calculation that the curvature tensor components are as follows:

$$
\begin{array}{llll}
R\left(e_{1}, e_{2}\right) e_{1} & =\alpha^{2} e_{2}-\alpha e_{1}, & R\left(e_{1}, e_{2}\right) e_{2}=-\alpha^{2} e_{1}+\alpha e_{3}, \\
R\left(e_{1}, e_{2}\right) e_{3}=0, & R\left(e_{1}, e_{3}\right) e_{1}=\alpha^{2} e_{3}, \\
R\left(e_{1}, e_{3}\right) e_{2}=\alpha e_{3}, & R\left(e_{1}, e_{3}\right) e_{3}=-\alpha^{2} e_{1}, \\
R\left(e_{2}, e_{3}\right) e_{1}=\alpha e_{3}, & R\left(e_{2}, e_{3}\right) e_{2}=\alpha^{2} e_{3}, \\
R\left(e_{2}, e_{3}\right) e_{3}=-\alpha^{2} e_{2} . & &
\end{array}
$$

It remains to prove that $d \eta=0$ and $d \Phi=2 \alpha \eta \wedge \Phi$. Also, it can be easily check that Nijenhuis torsion of $\phi$ is zero. By simple calculations, it is seen that $d \eta=0$. It follows that $\Phi\left(e_{1}, e_{2}\right)=1$ and $\Phi\left(e_{i}, e_{j}\right)=0$ for $i \leq j$. Thus the essential non-zero component of $\Phi$ is

Then we have

$$
\Phi\left(\frac{\partial}{\partial x_{1}}, \frac{\partial}{\partial x_{2}}\right)=\frac{1}{e^{2 \alpha x_{3}}},
$$

Therefore, the exterior derivative $d \Phi$ is given by

$$
\Phi=\frac{1}{e^{2 \alpha x_{3}}} d x_{1} \wedge d x_{2} .
$$

$$
d \Phi=-2 \alpha e^{-2 \alpha x_{3}} d x_{1} \wedge d x_{2} \wedge d x_{3} .
$$

Since $\eta=d x_{3}$, we obtain $d \Phi=2 \alpha \eta \wedge \Phi$. Consequently, $(\phi, \xi, \eta, g)$ is $\alpha$-cosymplectic structure for $\alpha \in \mathbb{R}$ and $e_{3}=\xi$. It can be noted that the structure is cosymplectic under the condition $\alpha=0$. Otherwise the structure is $\alpha$-Kenmotsu on $M^{3}$.

\section{ACKNOWLEDGEMENTS}

The author is grateful to the referee for valuable comments and suggestions.

\section{REFERENCES}

[1] Arslan, K., Murathan, C. and Ozgür, C.,On $\phi$-Conformally flat contact metric manifolds, Balkan J. Geom. Appl. (BJGA), 5 (2) (2000), 1-7.

[2] Arslan, K., Murathan, C. and Ozgür, C., On contact manifolds satisfying certain curvature conditions, Proceedings of the Centennial "G. Vranceanu" and the Annual Meeting of the Faculty of Mathematics (Bucharest, 2000). An. Univ. Bucureşti Mat. Inform., 49 (2) (2000), 17-26.

[3] Blair, D., Contact manifolds in Riemannian Geometry, Lecture Notes in Math. Springer-Verlag, Berlin-HeidelbergNew-York, 509 (1976).

[4] Cabrerizo, J.L, Fernandez, L.M., and Zhen, G., The structure of a class of K-contact manifolds, Acta Math. Hungar, 82 (4) (1999), 331-340.

[5] De, U.C., Binh,T.Q. and Shaikh, A.A., On weakly symmetric and weakly Ricci-symmetric K-contact manifolds, Acta Mathematica Acadeiae Paeda Gogicae Nyíregyháziensis, 16(2000), 65-71.

[6] Ishii, Y., On conharmonic transformations, Tensor N.S, 7 (1957), 73-80.

[7] Jun, J-B., De, U.C. and Pathak, G.,On Kenmotsu manifolds, J. Korean Math. Soc. 42(2005), No.3, 435-445.

[8] Kenmotsu, K., A class of contact Riemannian manifold, Tohoku Math. Jour. 24(1972), 93-103.

[9] Kim, T.W, Pak, H.K., Canonical foliations of certain classes of almost contact metric structures, Acta Math. Sinica, Eng. Ser. Aug., 21, 4 (2005), 841-846.

[10] Khan, Q, On conharmonically and special weakly Ricci-symmetric Sasakian manifolds, Novi Sad J. Math., 34(2004), $71-77$.

[11] Özgür,C.,On weak symmetries of Riemann para Sasakian manifolds, Radovi Matamatički,11(2002), $263-270$.

[12] Özgür,C., $\phi$-Conformally flat Lorentzian para-Sasakian manifolds, Radovi Mathematicki, 12(2003), 99-106.

[13] Singh, H., Khan, Q., On special weakly symmetric Riemannian manifolds. Publ. Math. Debrecen, Hungary 58/3(2001), 523-536.

[14] Tamássy, L. and Binh, T.Q., On weakly symmetric and weakly projective symmetric Riemannian manifolds, Coll. Math. Soc. J. Bolyai, 56(1992), 663-670.

[15] Tamássy, L. and Binh, T.Q., On weak symmetries of Einstein and Sasakian manifolds, Tensor N.S, 53(1993), $140-148$.

[16] Tanno, S., The automorphism groups of almost contact Riemannian manifolds, Tohoku Math. J., 21 (1969), 21-39.

[17] Yano K., Kon, M., Structures on Manifolds, Series in Pure Math, Vol 3, World Sci, 1984.

[18] Zhen, G., On conformally symmetric K-contact manifolds, Chinese Quart. J. of Math, 7 (1992), 5-10.

[19] Zhen, G., Cabrerizo, J.L., Fernandez L.M. and Fernandez, M., On $\xi$-conformally flat contact metric manifolds, Indian J. Pure Appl. Math, 28 (1997), 725-734. 